\title{
Identification of Cherry Leaf Disease Infected by Podosphaera Pannosa via Convolutional Neural Network
}

\author{
Keke Zhang, College of Engineering, Northeast Agricultural University, Harbin, China \\ Lei Zhang, Department of Radiology, University of Pittsburgh, Pittsburgh, USA \\ Qiufeng Wu, College of Science, Northeast Agricultural University, Harbin, China
}

\begin{abstract}
The cherry leaves infected by Podosphaera pannosa will suffer powdery mildew, which is a serious disease threatening the cherry production industry. In order to identify the diseased cherry leaves in early stage, the authors formulate the cherry leaf disease infected identification as a classification problem and propose a fully automatic identification method based on convolutional neural network (CNN). The GoogLeNet is used as backbone of the CNN. Then, transferred learning techniques are applied to fine-tune the CNN from pre-trained GoogLeNet on ImageNet dataset. This article compares the proposed method against three traditional machine learning methods i.e., support vector machine (SVM), k-nearest neighbor (KNN) and back propagation (BP) neural network. Quantitative evaluations conducted on a data set of 1,200 images collected by smart phones, demonstrates that the $\mathrm{CNN}$ achieves best precise performance in identifying diseased cherry leaves, with the testing accuracy of $99.6 \%$. Thus, a CNN can be used effectively in identifying the diseased cherry leaves.
\end{abstract}

\section{KEYWORDS}

Cherry leaf, Convolutional neural network, Deep learning, Podosphaera pannosa, Transfer learning

\section{INTRODUCTION}

Podosphaera pannosa (syn. Sphaerotheca pannosa) is a fungus which causes powdery mildew in various farm and greenhouse crops worldwide (leus et al., 2006). Powdery mildew is a very common disease on many types of plant such as apricot, peach, plum, roses, cherry. Typical symptoms of leaves infected by powdery mildew are covered by white powdery fungal, and turned out distortion, scrappy and premature defoliation subsequently (Shetty et al., 2012). Powdery mildew causes severe yield and quality reduction; therefore, it is urgently needed to utilize an effective method to diagnose the powdery mildew in early stage.

There are several approaches of diagnosing plant diseases. The first method is the traditional pathology way, that is, observing disease, obviously it is an enormous workload, time-consuming and highly rely on the plant pathologist. In response to this issue, the Enzyme-linked Immunosorbent Assay (ELISA) is proposed, which can detect the viral protein content of plant extract (Clark et al., 1980). However, it is hardly effective in diagnosing fungal disease and bacterial disease. Furthermore, the real-time polymerase chain reaction (PCR) method is utilized in testing plant pathogen (Schaad et al., 2002), the method is superior to the two aforementioned methods in speed and accuracy, but it is difficultly to implement widely, since the operator should possess professional skill, and the most

This article, originally published under IGI Global's copyright on April 1, 2019 will proceed with publication as an Open Access article starting on February 4, 2021 in the gold Open Access journal, International Journal of Agricultural and Environmental Information Systems (converted to gold Open Access January 1, 2021), and will be distributed under the terms of the Creative Commons Attribution License (http://creativecommons.org/licenses/by/4.0/) which permits unrestricted use, distribution, and production in any medium, provided the 
important reason is that the equipment utilized is very expensive. Thus, we propose an image-based diagnosing method via machine learning, which is real-time, high accuracy, strong operability, and can be potentially used in farm.

Utilizing image-based machine learning algorithm to identify plant disease can be formulated as image classification problem, image classification algorithms are usually divided into feature extraction and classification (Yan et al., 2016), that is, extracting features by suitable feature extractor, and then build a classifier by the extracted features (Lin et al., 2016). A type of the machine learning which is called supervised learning has been widely used in classification problem, which means features are attached correctly labels before sent into classifier. The typical process is that, sending training set which is consisted of features and labels to a learning algorithm which is a hypothesis function. Thus, we can get the prediction according to features fed into the hypothesis function. Traditional supervised learning algorithms have been applied to identifying plant disease, i.e., utilizing Support Vector Machine(SVM) to detect little leaf disease in pine trees in United States (Singh et al., 2017), refining the prevalence of wheat scab according to Back Propagation(BP) neural network (Jin et al., 2012), classifying huanglongbing and citrus canker infected leaves by K-Nearest Neighbor(KNN) (Sankaran et al., 2013), and detecting plant leaf disease by Probabilistic Neural Network(PNN) (Stephen et al., 2017).

In fact, the conventional machine learning exists several shortages in image classification problem, image classification is usually divided into feature extraction and classification (Yan et al., 2016), both feature extractor and classifier are hard to correctly select in specific problem. Thus, deep learning is proposed to overcome disadvantages mentioned above, it is composed of multiple processing layers to representation of data with multiple levels of abstraction (Yann et al., 2015). In addition, computer vision, medical imaging, and signal processing tasks have evidently showcased the effectiveness of deep features learned by deep neural networks (Zhou et al., 2017; Liu et al., 2015; Ouyang et al., 2013; Yan et al., 2016; Melendez et al., 2015; Zhang et al., 2016; Xie et al., 2017; Luo et al., 2016; Luo et al., 2017) which are likely to replace the conventional hand-crafted features (Yann et al., 2015). Meanwhile, deep learning has been initially used in agriculture, such as plant species identification (Mehdipour et al., 2017), weed identification (Tang et al., 2017), blood defects in cod fillets classification (Misimi et al., 2017), and pest identification (Cheng et al., 2017).

This paper focus on identifying cherry leaf infected by Podosphaera pannosa with deep Convolutional Neural Network (CNN). The rest of this paper is organized as follows. Section 2 introduces the dataset of cherry leaf and the GoogLeNet. Section 3 displays the three comparative experiments between $\mathrm{CNN}$ and the state-of-the-art. Section 4 concludes this paper.

\section{MATERIALS AND METHODS}

The cherry leaf infected by Podosphaera pannosa will suffer from powdery mildew, thus, identifying cherry leaf infected by Podosphaera pannosa only need to identify the cherry leaf is healthy or diseased, which can be formulated as a binary classification problem. The classification process of this work can be described as follows. A pair $\left(x^{(i)}, y^{(i)}\right)$ is called a training sample(i.e., an input cherry leaf image), the dataset that we will be used to learn is a list of $m$ training samples $\left\{\left(x^{(i)}, y^{(i)}\right) ; i=1, \ldots, m\right\}$, which is called a training set (See Table 2). Then make the training set to learn a function $h: X \mapsto Y$ ( $X$ denote the space of input values, $Y$ denotes the space of output values), thus, $h(x)$ can be considered as a suitable predictor, that is, a classifier (i.e., CNN). So, we can obtain a series of discrete values (i.e., predict labels, "Health" and "Podosphaera pannosa") from the classifier.

$\mathrm{CNN}$ is mainly stacked by three types of layers, i.e., convolutional layer, pooling layer and fullyconnected layer. $\mathrm{CNN}$ has several excellent properties. Firstly, CNN can combine feature extraction with classification (Anthimopoulos et al., 2016), that is, the proceed of classification algorithm is 
not needed to divide into feature extraction and classification, CNN will extract feature and select classifier automatically. In addition, it is not necessary to carry on a preprocessing step to separate the target things from a complex background image, owing to CNN can find image features by itself (Dyrmann et al., 2016). Furthermore, the weights of convolutional layer are shared, according to utilizing a same filter to slide over all image spatial locations, parameters are dramatically reduced as well, and it largely improves the efficiency of CNN.

The classification process by CNN can be represented with the following formula. Firstly, send the training samples (i.e., training cherry leaves images) to the classifier (i.e., CNN), then, convolution process is conducted, that is, a series of filters slide over the feature map of the previous layer, and the weight matrices do dot product.

$$
x_{j}^{l}=f\left(\sum_{i \in N_{j}} x_{i}^{l-1} * w_{j}^{l}+b_{j}^{l}\right)
$$

Where $f(\cdot)$ is activation function, typically a rectified linear activation ReLU:

$$
f(x)=\max (x, 0)
$$

$N_{j}$ is the number of filters of the certain layer, $x_{i}^{l-1}$ represents the feature map of the previous layer, $w_{j}^{l}$ is the weight matrix, and $b_{j}^{l}$ is the bias term.

Following convolution operation, max pooling or average pooling methods are carried out. Furthermore, the learned features are sent to fully connected layer.

$$
x_{j}^{l}=\operatorname{squash}\left(x_{i}^{l-1}\right)
$$

Where $\operatorname{squash}(\cdot)$ is a compression function, the function compress the learned features to a vector.

The softmax regression often follows the final fully connected layer, an input $x$ will get the probability of belong to class $i$.

$p(y=i \mid x ; \theta)=\frac{e^{\theta_{i}^{T} x}}{\sum_{j=1}^{k} e^{\theta_{i}^{T} x}}$

Where $y$ is the response variable (i.e., predict label), $k$ is the number of categories, $\theta$ is the parameters of our model.

In this work, transfer learning is used to fine-tune the GoogLeNet. GoogLeNet is the first place of the ImageNet of Large-Scale Visual Recognition Challenge (ILSVRC) in 2014, which is trained from 1.2 million images, and classify 100000 testing images into 1000 leaf-node categories (Szegedy et al., 2015). Transfer learning in GoogLeNet is fine-tune the weights from the cherry leaf dataset, using three new layers, a fully connected layer, a softmax layer and a classification output layer replace the last three layers of the network, setting the size of the final connected layer to 2 , which is equal to the number of classification category in this work. It should be noted that the size of input images must be shaped into $224 \times 224$, which satisfy the input pixel size requirement of GoogLeNet. 
Figure 1. Cherry leaf image dataset. The first row shows cherry leaves infected by Podosphaera pannosa and the second row of the figure displays healthy cherry leaves.

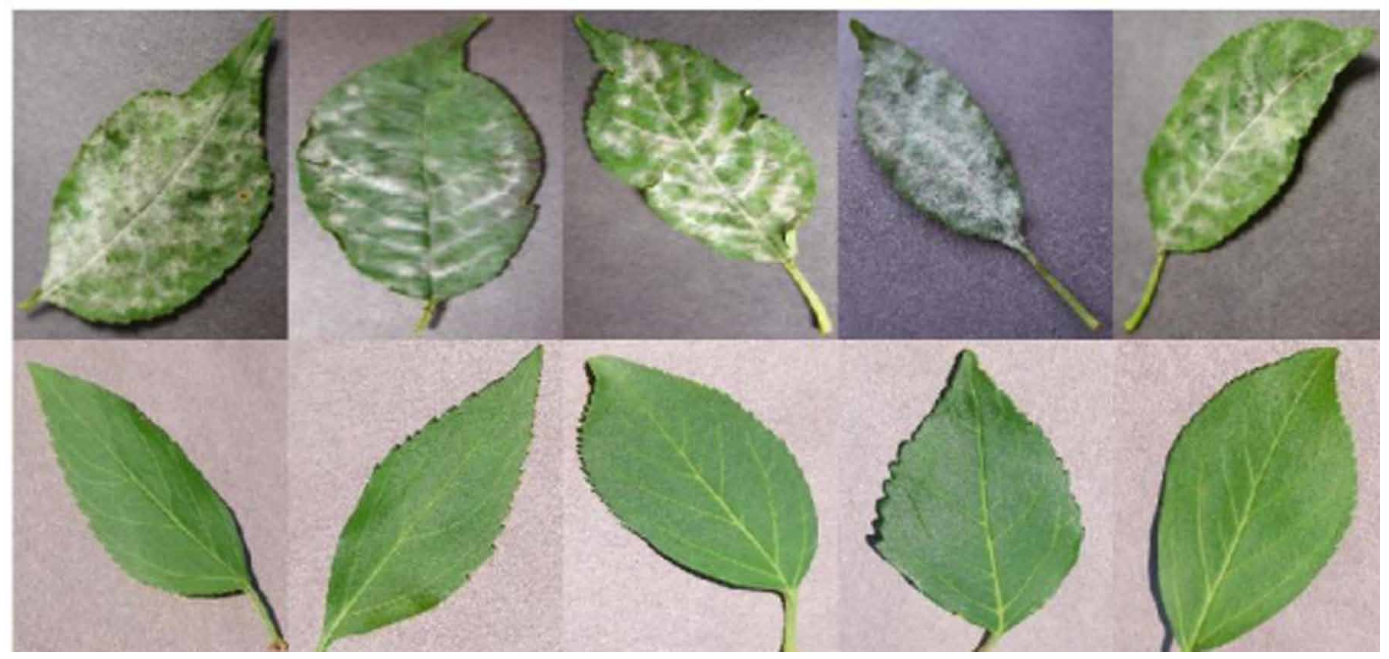

The performance of the fine-tuned GoogLeNet is evaluated by Receiver Operating Characteristic (ROC) curve and Area Under ROC Curve (AUC), and three comparative experiments are carried out between CNN and the state-of-the-art such as Support Vector Machine (SVM), K-Nearest Neighbor (KNN) and Back Propagation (BP) Neural Network (Liu et al., 2013; Xu et al., 2013).

This section displays the utilized dataset in this work, and introduces the GoogLeNet. Meanwhile, feature visualization of the trained $\mathrm{CNN}$ is conducted.

\subsection{Dataset}

This paper aims at identifying cherry leaf infected by Podosphaera pannosa, the dataset which contains healthy cherry leaf and diseased leaf infected by Podosphaera pannosa comes from an open access repository of images (Hughes and Salathé, 2015), the infected cherry leaves will suffer from powdery mildew and the symptom of the diseased leaves shows that leaves are covered by white powdery fungal, meanwhile often accompanied by curly and dry (See the first row of Figure 1) (Shetty et al., 2012). The first row of Figure 1 displays the diseased leaves infected by Podosphaera pannosa, and the second row shows the healthy cherry leaves. The statistics of dataset is shown in Table 1, the number of healthy cherry leaves is 600 , which is equal to the number of diseased cherry leaves.

\subsection{GoogLeNet}

GoogLeNet is a deeper network with computational efficiency, which has 22 layers, 12 times fewer parameters than AlexNet which contains 60 million parameters (Szegedy et al., 2016). The critical part of the architecture of GoogLeNet is Inception module (See Figure 2), which contains four 1x 1 convolutional layers, one $3 \times 3$ convolutional layer, one $5 \times 5$ convolutional layer and one $3 \times 3$ maxpooling layer. The whole architecture of GoogLeNet is stacked by Inception modules on top of each other. The codenamed Inception is inspired by the network in network (Lin et al., 2014), the most obvious difference between the naive Inception module and the Inception module given in Figure 2 is that, additional $1 \times 1$ convolutional layers are added to the Inception module. The additional $1 \times 1$ convolutional layer not only reduces the spatial dimension largely, but also limits the size of GoogLeNet (Szegedy et al., 2015). The fine-tuned GoogLeNet in this work is removed the last three layers, and add three new layers includes one fully connected layer, one softmax layer and one classification layer. 
Table 1. Setup in Cherry leaf Image Dataset

\begin{tabular}{|l|l|}
\hline \multicolumn{1}{|c|}{ Category } & \multicolumn{1}{c|}{ Sample } \\
\hline Health & 600 \\
\hline Podosphaera pannosa & 600 \\
\hline
\end{tabular}

Meanwhile, the size of fully connected layer is modified to 2, since identify cherry leaves infected by Podosphaera pannosa is a binary classification in this paper.

\subsection{Feature Visualization}

We examined the features of the first convolutional layer and the final fully connected layer learned of the CNN by utilizing the t-distributed Stochastic Neighbour Embedding (t-SNE) algorithm (Maaten and Hinton, 2008) (See Figure3). 240 test images were used to extract the features, blue points represent the cherry leaves infected by Podosphaera pannosa, and red points represent the healthy cherry leaves, respectively. Figure 3 (a) displays the scatterplot of the features at the first convolutional layer of CNN. Obviously, blue points and red points are mixed together, since the features of the input samples could not be used to differentiation the two different samples properly. Figure3 (b) shows the scatterplot at the final fully connected layer of $\mathrm{CNN}$, blue points and red points are separated distinctly, which indicates that the features learned with layered structure of the CNN can be used to classify the cherry leaves precisely. Both Figure 3 (a) and Figure 3(b) indicate that the end-to-end learning mechanism of the $\mathrm{CNN}$ can efficiently separate the two different classes of leaves.

\section{EXPERIMENTAL RESULTS}

This section shows the setup of experiment, furthermore, the comparative experiment on CNN and the state-of-the-art is shown in detail.

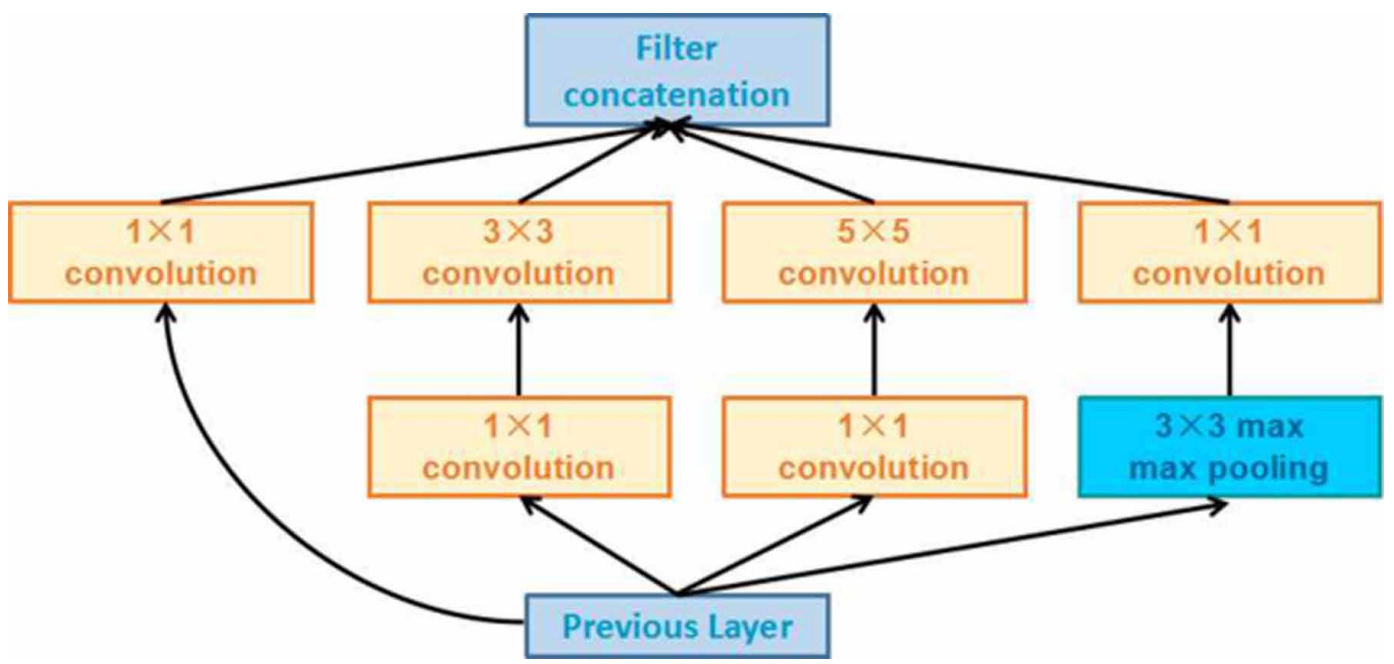


Figure 3. Two-dimensional scatterplots of high-dimensional features generated with t-SNE. (a) Scatterplot at the first convolutional layer of CNN. (b) Scatterplot at the final fully connected layer of CNN.
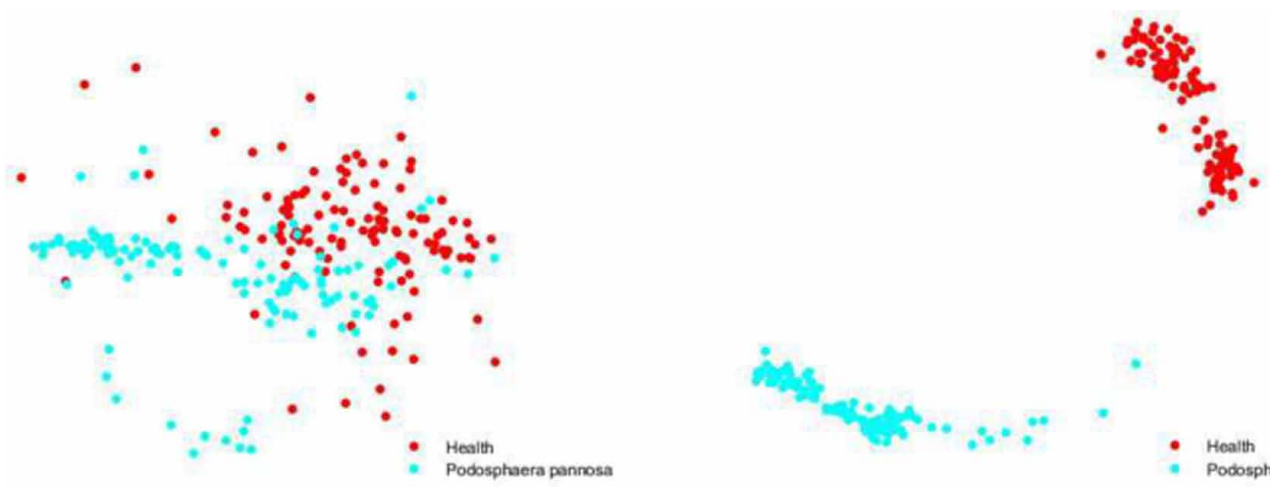

\subsection{Experimental Setup}

The dataset utilized in this paper includes 600 healthy cherry leaves and 600 diseased leaves infected by Podosphaera pannosa (See Table1), and it comes from an open access repository of images on Plant Village (Liu et al., 2013). The dataset is processed into two types, the first type dataset is consisted of $80 \%$ training samples and $20 \%$ testing samples, which is called type1 (See Table2). In addition, 5 -folder cross validation is used. The cross-validation dataset is composed of training samples and validation samples, both them are subsets of 960 training samples (See Table2), it is called cvtype1, cvtype 2 and so on.

For the purpose of evaluating classification performance of CNN and the state-of-the-art, three comparative experiments are carried out. Utilizing CNN to identify diseased cherry leaves has only one stage, and the notation is CNN. The traditional machine learning is generally divided into feature extraction and classification two stages. In feature extraction stage, 100 features are extracted from each cherry leaf image using the Scale-Invariant Feature Transform (SIFT) algorithm (Li et al., 2011; Lazebnik et al., 2006). In classification stage, the extracted features are delivered into different

Table 2. Details of dataset

\begin{tabular}{|c|c|c|c|c|}
\hline \multirow{2}{*}{ dataset } & subset & Health & Podosphaera pannosa & overall \\
\hline \multirow{3}{*}{ type1 } & training samples & 480 & 480 & 960 \\
\cline { 2 - 5 } & testing samples & 120 & 120 & 240 \\
\hline \multirow{3}{*}{ cvtype1 } & training samples & 380 & 388 & 768 \\
\cline { 2 - 5 } & validation samples & 100 & 92 & 192 \\
\hline \multirow{3}{*}{ cvtype3 } & training samples & 385 & 383 & 768 \\
\cline { 2 - 5 } & validation samples & 95 & 97 & 192 \\
\hline \multirow{2}{*}{ cvtype4 } & training samples & 388 & 380 & 768 \\
\cline { 2 - 5 } & training samples & 92 & 100 & 768 \\
\hline \multirow{2}{*}{ cvalidation samples } & training samples & 99 & 387 & 192 \\
\cline { 2 - 5 } & validation samples & 94 & 93 & 768 \\
\hline
\end{tabular}


classifiers i.e., SVM, KNN, and BP. Combining SIFT with SVM, KNN, and BP are referred to as SVM, KNN, and BP, respectively. The first experiment presents the testing classification accuracy of $\mathrm{CNN}$ and the state-of-the-art (See Figure 4). Meanwhile, classification results per class for CNN and the state-of-the-art is presented (See Figure 5), the evaluation metric is classification accuracy as well. The third comparative experiment focus on comparing the overall performance of each classification algorithm, by plotting the ROC curves of CNN and the state-of-the-art. In addition, the AUC values are computed (See Figure 6). The final experiment is to execute 5-folder cross validation, and carry out statistical significance analysis (See Table 3).

\subsection{Comparative Experiments on $\mathrm{CNN}$ and the State-of-the-Art}

The first comparison metric is accuracy:

$$
\text { accuracy }=\frac{\text { true positives }+ \text { true negatives }}{\text { positives }+ \text { negatives }}
$$

The "true positives" is the number of correctly predicted "Health" label, "positives" is the number of "Health" label, "true negatives" is the number of correctly predicted "Podosphaera pannosa" label, and "negatives" is the number of "Podosphaera pannosa" label. The testing accuracies is reported in Figure 4, each bar represents the testing accuracy of different classification method. It is clearly that the $\mathrm{CNN}$ achieves the best performance, with the accuracy of $99.6 \%$. In addition, the classification results per class for four methods is presented in Figure 5. The notation 'Health' and 'Podosphaera pannosa' represent two classes in this work, that is, health cherry leaves and the diseased cherry leaves infected by Podosphaera pannosa. The bar with red color represents the accuracy of "Health" category, and the bar with blue color represents the accuracy of "Podosphaera pannosa" category, respectively. The best performance, "Health" classification accuracy of $100 \%$ and "Podosphaera pannosa" classification accuracy of $99.2 \%$, is achieved by CNN. Thus, it can be considered that the CNN has the best accuracy in identifying cherry leaves infected by Podosphaera pannosa.

Figure 6 displays the ROC curves of $\mathrm{CNN}$ and the state-of-the-art. ROC curves have been widely used in machine learning field as an overall performance evaluation index (Anthimopoulos et al., 2016; Swets et al., 2000), since the ROC curve is scarcely affected by the skewed and the changes of class distribution (Fawcett, 2006). The $X$ axis is "False positive rate"(i.e., fp rate, 1-specificity), the $Y$ axis is "True positive rate"(i.e., tp rate, also called sensitivity or recall),

$$
\begin{aligned}
\text { tp rate } & =\frac{\text { positives correctly classified }}{\text { total positives }} \\
f p \text { rate } & =\frac{\text { negatives incorrectly classified }}{\text { total negatives }}
\end{aligned}
$$

"positives" represents "health" label, and "negatives" represents "Podosphaera pannosa" label. At the same time, the AUC values of each ROC curve are given in Figure 6. In Figure 6, the ROC curve of $\mathrm{CNN}$ presents an upper left triangle straight line, that is, CNN achieves the excellent performance, with the AUC of 1 . It is proved again that the $\mathrm{CNN}$ is superior to the state-of-the-art in classifying cherry leaves infected by Podosphaera pannosa.

The test of significance experiment is implemented with AUC values from 5-folder cross validation, in order to detect whether there is a significant difference between CNN and the stateof-the-art. The level of significance is set to 0.05 , which means that the comparative methods exist statistically significance difference when the p-value is less than 0.05 . Table 3 lists the results of 


\section{Accuracy}

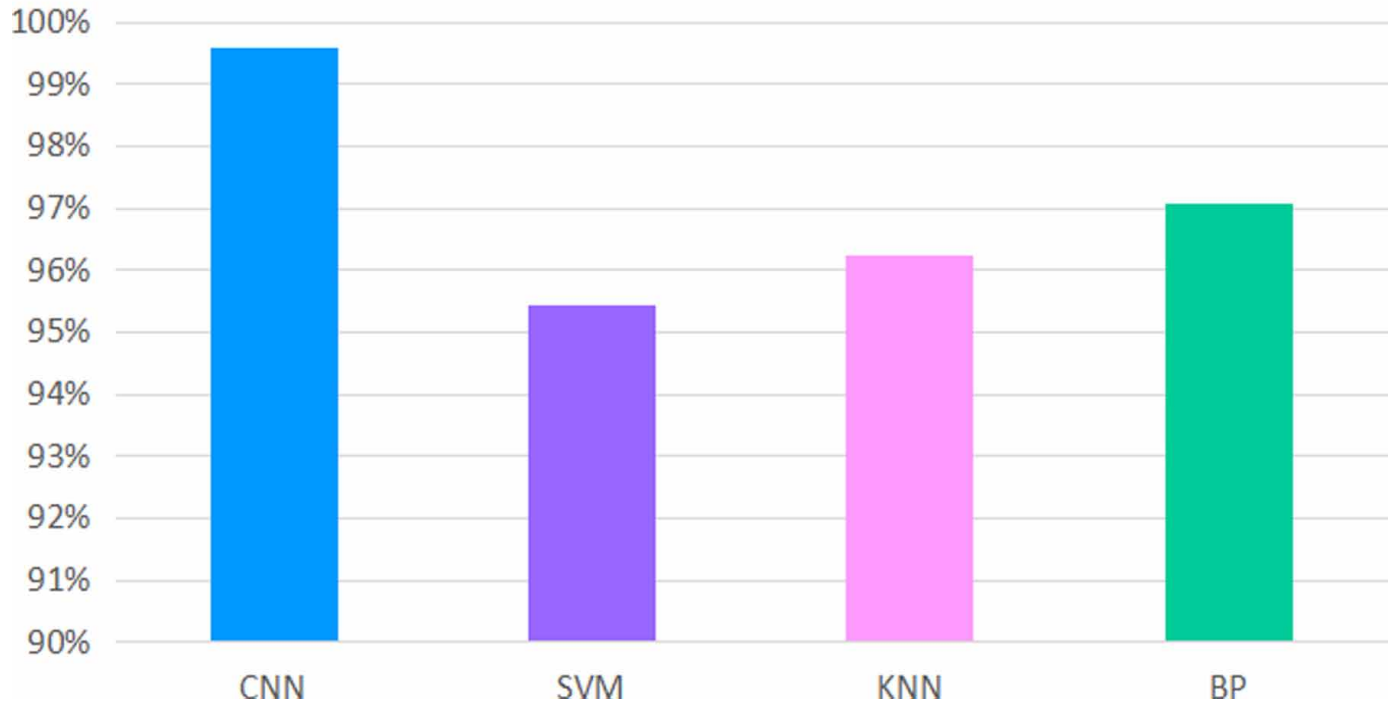

significance experiment. The AUC column comes from the average AUC values from 5-folder cross validation, meanwhile, $\mathrm{p}$-values which are obtained from several significance experiments is given. The p-values of CNN vs.SVM is $0.0193, \mathrm{CNN}$ vs. $\mathrm{KNN}$ is 0.0094 and $\mathrm{CNN}$ vs. BP is 0.0316 , It is clearly that all three p-values are less than 0.05 . Therefore, we can draw a conclusion that the difference is significant between the $\mathrm{CNN}$ and the state-of-the-art.

Figure 5. Classification results per class for four methods

\section{Accuracy}

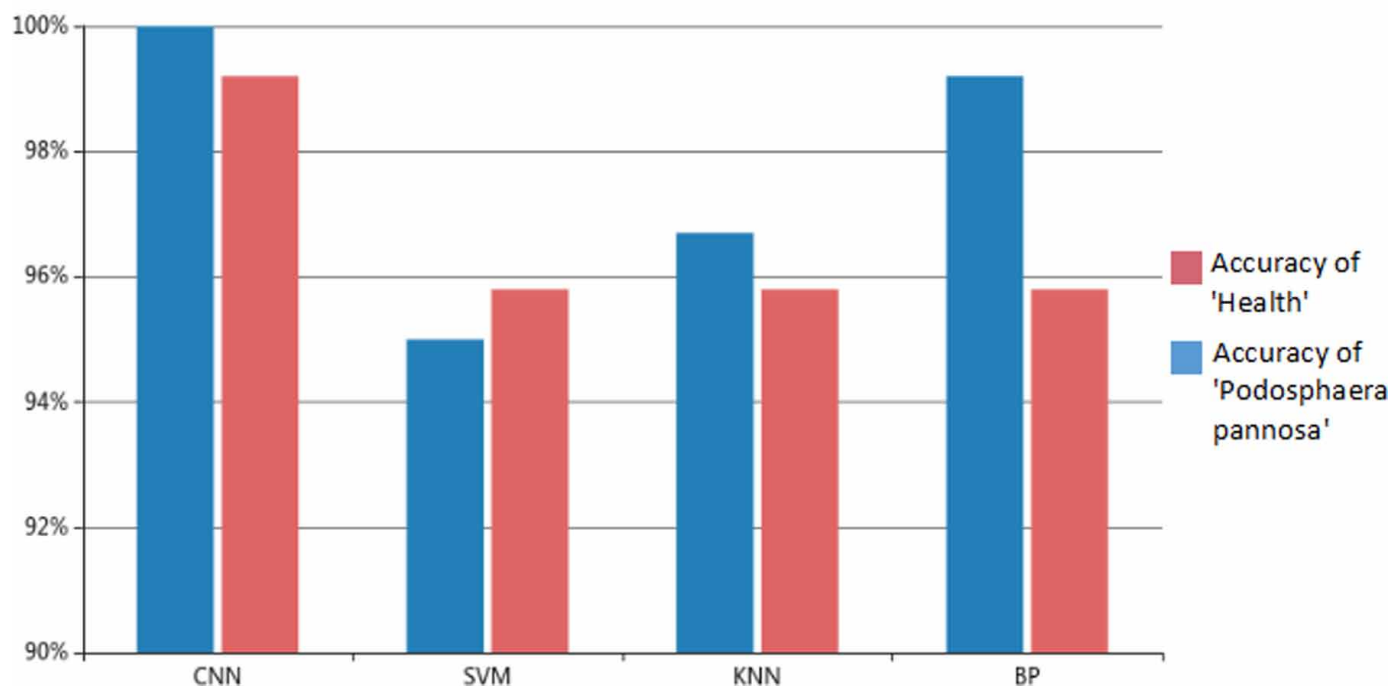




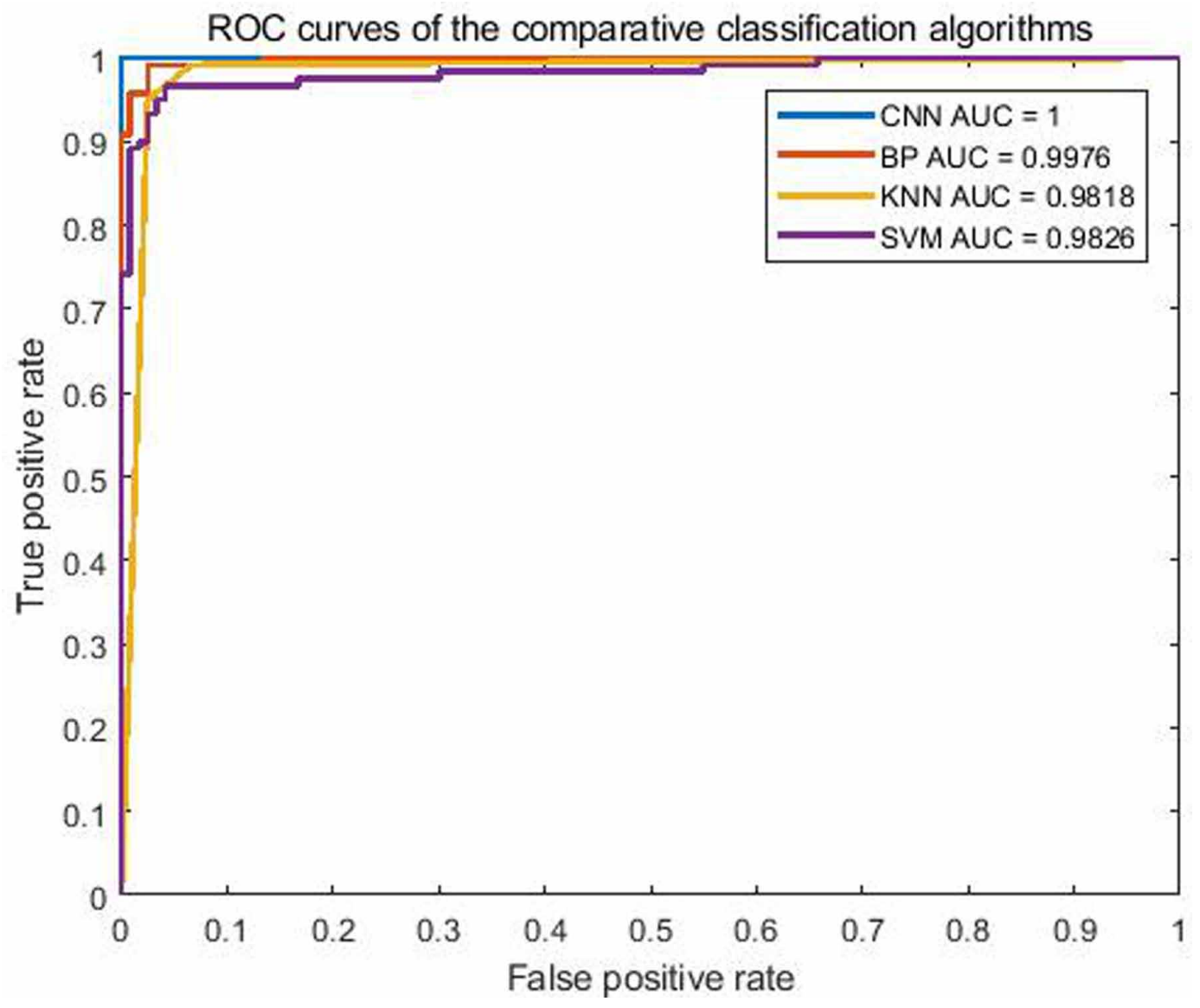

\section{CONCLUSION}

In this paper, a novel deep-learning based approach for identifying cherry leaves infected by Podosphaera pannosa has been presented.which owns the end-to-end learning mechanism. Compared with the traditional machine learning, which suffer from selecting appropriate feature extractor and classifier, the proposed method learning the feature and the classifier jointly by an end-to-end learning mechanism. The proposed method is compared against three conventional machine learning methods (i.e., SVM, KNN, and BP) on three different experiments. The testing accuracy and the classification

Table 3. Detection Performance (AUC) with different methods on the 5 -folder cross validation and the p-values obtained when comparing with different methods

\begin{tabular}{|c|c|c|c|c|c|}
\hline method & AUC & vs.SVM & vs.KNN & vs.BP & vs.CNN \\
\hline SVM & 0.9840 & -- & 0.3918 & 0.0334 & $\mathbf{0 . 0 1 9 3}$ \\
\hline KNN & 0.9883 & 0.3918 & -- & 0.0280 & $\mathbf{0 . 0 0 9 4}$ \\
\hline BP & 0.9983 & 0.0334 & 0.0280 & -- & $\mathbf{0 . 0 3 1 6}$ \\
\hline CNN & 1 & $\mathbf{0 . 0 1 9 3}$ & $\mathbf{0 . 0 0 9 4}$ & $\mathbf{0 . 0 3 1 6}$ & -- \\
\hline
\end{tabular}


results per class for four methods indicate that the high precision performance of $\mathrm{CNN}$, with the testing accuracy of $99.6 \%$ and 'Health' classification accuracy of $100 \%$ respectively. In addition, ROC curves and AUC values were presented, which indicates the best performance of the proposed method. It conveys that the overall performance of $\mathrm{CNN}$ is superior to the state-of-the-art. Furthermore, the significance test shows that the CNN is significantly superior to the other three methods, with the p-values of 0.0193, 0.0094 and 0.0316, according to comparing with SVM, KNN and BP respectively. To our knowledge, this is the first study which applies the deep-learning based method to the cherry leaf disease identification problem. The practical study of this work can be easily extended to other plant leaf disease identification problem.

\section{ACKNOWLEDGEMENT}

This study was supported by Public Welfare Industry (Agriculture) Research Projects Level-2 (201503116-04-06), Student Innovation Practical Training (201710224041), Postdoctoral Foundation of Heilongjiang Province (LBHZ15020), Harbin Applied Technology Research and Development Program (2017RAQXJ096), Economic Decision Making and Early Warning of Soybean Industry in Technology Collaborative Innovation System of Soybean Industry in Heilongjiang Province (201704). Qiufeng $\mathrm{Wu}$ is the corresponding author for this article. 


\section{REFERENCES}

Leus, L., Dewitte, A., Huylenbroeck, J., Vanhoutte, N., Bockstaele, E., \& Höfte, M. (2006). Podosphaera pannosa (syn. Sphaerotheca pannosa) on Rosa and Prunus spp.: Characterization of pathotypes by differential plant reactions and ITS sequences. Journal of Phytopathology, 154(1), 23-28. doi:10.1111/j.1439-0434.2005.01053.x

Shetty, R., Jensen, B., Shetty, N. P., Hansen, M., Hansen, C. W., Starkey, K. R., \& Jørgensen, H. J. L. (2012). Silicon induced resistance against powdery mildew of roses caused by Podosphaera pannosa. Plant Pathology, 61(1), 120-131. doi:10.1111/j.1365-3059.2011.02493.x

Clark, D. P., \& Dougherty, R. M. (1980). Detection of avian oncovirus group-specific antigens by the enzymelinked immunosorbent assay. The Journal of General Virology, 47(2), 283-291. doi:10.1099/0022-1317-47-2283 PMID:6245172

Schaad, N. W., \& Frederick, R. D. (2002). Real-time PCR and its application for rapid plant disease diagnostics. Canadian Journal of Plant Pathology, 24(3), 250-258. doi:10.1080/07060660209507006

Yan, Z., Zhan, Y., Peng, Z., Liao, S., Shinagawa, Y., Zhang, S., \& Zhou, X. S. et al. (2016). Multi-instance deep learning: Discover discriminative local anatomies for bodypart recognition. IEEE Transactions on Medical Imaging, 35(5), 1332-1343. doi:10.1109/TMI.2016.2524985 PMID:26863652

Guan, L., Liu, Z. Z., Wu, Q. F., \& Wang, L. L. (2016). Multi-type Feature Fusion Technique for Weed Identification in Cotton Fields. International Journal of Signal Processing Image Processing and Pattern Recognition., 9(2), 355-368. doi:10.14257/ijsip.2016.9.2.30

Singh, V., \& Misra, A. K. (2017). Detection of plant leaf diseases using image segmentation and soft computing techniques. Information Processing in Agriculture, 4(1), 41-49. doi:10.1016/j.inpa.2016.10.005

Liu, K., Guo, W. Y., Shen, X. L., \& Tan, Z. F. (2012). Research on the Forecast Model of Electricity Power Industry Loan Based on GA-BP Neural Network. Energy Procedia, 14, 1918-1924. doi:10.1016/j.egypro.2011.12.1188

Sankaran, S., \& Ehsani, R. (2013). Comparison of visible-near infrared and mid-infrared spectroscopy for classification of Huanglongbing and citrus canker infected leaves. Agricultural Engineering International: CIGR Journal, 15(3), 75-79.

Stephen, G. W., Forrest, S. B., Eric, Y. X., Wang, Y. X., Chang, Y. F., \& Xiang, Q. L. (2007). A Leaf Recognition Algorithm for Plant Classification Using Probabilistic Neural Network. In IEEE International Symposium on Signal Processing and Information Technology (pp. 11-16).

Yann, L., Yoshua, B., \& Geoffrey, H. (2015). Deep Learning. Nature, 521(7553), 436-444. doi:10.1038/ nature14539 PMID:26017442

Zhou, Z. W., Shin, J., Zhang, L., Gurudu, S., Gotway, M., \& Liang, J. M. (2017). Fine-tuning convolutional neural networks for biomedical image analysis. In Actively and Incrementally. IEEE Conference on Computer Vision and Pattern Recognition (pp. 7340-7349).

Liu, Z.W., Luo, P., Wang, X.G. \& Tang, X.O. (2015). Deep Learning Face Attributes in the Wild. In 2015 IEEE International Conference on Computer Vision (ICCV) (pp. 3730-3738). doi:10.1109/ICCV.2015.425

Ouyang, W. L., \& Wang, X. G. (2013). Joint Deep Learning for Pedestrian Detection. In IEEE International Conference on Computer Vision (pp. 2056-2063).

Yan, Z. N., Zhan, Y. Q., Peng, Z. G., Liao, S., Shinagawa, Y., Zhang, S. T., \& Zhou, X. S. et al. (2016). MultiInstance Deep Learning: Discover Discriminative Local Anatomies for Bodypart Recognition. IEEE Transactions on Medical Imaging, 35(5), 1332-1343. doi:10.1109/TMI.2016.2524985 PMID:26863652

Melendez, J., van Ginneken, B., Maduskar, P., Philipsen, R. H., Reither, K., Breuninger, M., \& Sanchez, C. I. et al. (2015). A novel multiple-instance learning-based approach to computer-aided detection of tuberculosis on chest X-rays. IEEE Transactions on Medical Imaging, 34(1), 179-192. doi:10.1109/TMI.2014.2350539 PMID:25163057

Zhang, L., Yang, F., Zhang, Y. D., \& Zhu, Y. J. (2016). Road crack detection using deep convolutional neural network. In IEEE International Conference on Image Processing (pp. 3708-3712). doi:10.1109/ICIP.2016.7533052 
Xie, D., Zhang, L., \& Bai, L. (2017). Deep learning in visual computing and signal processing. Applied Computational Intelligence and Soft Computing, 1-13. doi:10.1155/2017/1320780

Luo, G. N., An, R., Wang, K. Q., Dong, S. Y., \& Zhang, H. G. (2016). A deep learning network for right ventricle segmentation in short-axis MRI. In Computing in Cardiology Conference (pp. 485-488). doi:10.23919/ CIC.2016.7868785

Luo, G., Dong, S., Wang, K., Zuo, W., Cao, S., \& Zhang, H. (2017). Multi-views Fusion CNN for Left Ventricular Volumes Estimation on Cardiac MR Images. IEEE Transactions on Biomedical Engineering. doi:10.1109/ TBME.2017.2762762 PMID:29035205

Mehdipour, G. M., Yanikoglu, B., \& Aptoula, E. (2017). Plant identification using deep neural networks via optimization of transfer learning parameters. Neurocomputing, 235, 228-235. doi:10.1016/j.neucom.2017.01.018

Tang, J. L., Wang, D., Zhang, Z. G., He, L. J., Xin, J., \& Xu, Y. (2017). Weed identification based on K-means feature learning combined with convolutional neural network. Computers and Electronics in Agriculture, 135, 63-70. doi:10.1016/j.compag.2017.01.001

Misimi, E., Øye, E. R., Sture, Ø., \& Mathiassen, J. R. (2017). Robust classification approach for segmentation of blood defects in cod fillets based on deep convolutional neural networks and support vector machines and calculation of gripper vectors for robotic processing. Computers and Electronics in Agriculture, 139, 138-152. doi:10.1016/j.compag.2017.05.021

Cheng, X., Zhang, Y. H., Chen, Y. Q., Wu, Y. Z., \& Yue, Y. (2017). Pest identification via deep residual learning in complex background. Computers and Electronics in Agriculture, 141, 351-356. doi:10.1016/j. compag.2017.08.005

Anthimopoulos, M., Christodoulidis, S., Ebner, L., Christe, A., \& Mougiakakou, S. (2016). Lung Pattern Classification for Interstitial Lung Diseases Using a Deep Convolutional Neural Network. IEEE Transactions on Medical Imaging, 35(5), 1207-1216. doi:10.1109/TMI.2016.2535865 PMID:26955021

Dyrmann, M., Karstoft, H., \& Midtiby, H. S. (2016). Plant species classification using deep convolutional neural network. Biosystems Engineering, 151, 72-80. doi:10.1016/j.biosystemseng.2016.08.024

Szegedy, C., Liu, W., Jia, Y. Q., Sermanet, P., Reed, S., Anguelov, D., \& Rabinovich, A. et al. 2015. Going deeper with convolutions. In Proceedings of the IEEE conference on computer vision and pattern recognition (pp. 1-9).

Liu, Z. Z., Xu, X. Y., \& Wu, Q. F. (2013). A Novel K-Nearest Neighbor Algorithm Based on I-divergence Criterion. ICIC Express Letters. Part B, Applications, 4(2), 243-248.

Xu, X. Y., Liu, Z. Z., \& Wu, Q. F. (2013). A Novel K-Nearest Neighbor Classification Algorithm Based on Maximum Entropy. International Journal of Advancements in Computing Technology, 5(5), 966-973. doi:10.4156/ijact.vol5.issue5.115

Hughes, D. P., \& Salathé, M. (2015). An open access repository of images on plant health to enable the development of mobile disease diagnostics. Retrieved from http://arxiv.org/abs/1511.08060

Szegedy, C., Vanhoucke, V., Ioffe, S., Shlens, J., \& Wojna, Z. 2016. Rethinking the inception architecture for computer vision. In Proceedings of the IEEE Conference on Computer Vision and Pattern Recognition (pp. 2818-2826). doi:10.1109/CVPR.2016.308

Lin, M., Chen, Q., \& Yan, S. (2014). Network in network. Computing Research Repository. arXiv:1312.4400

Maaten, L., \& Hinton, G. (2008). Visualizing data using t-SNE. Journal of Machine Learning Research, 9 , 2579-2605.

Li, P., Ma, J., \& Gao, S. (2011). Actions in still web images: visualization, detection and retrieval. In International Conference on Web-Age Information Management (pp. 302-313). doi:10.1007/978-3-642-23535-1_27

Lazebnik, S., Schmid, C., \& Ponce, J. (2006). Beyond bags of features: Spatial pyramid matching for recognizing natural scene categories. In IEEE computer society conference on Computer vision and pattern recognition (Vol. 2, pp. 2169-2178). 
Anthimopoulos, M., Christodoulidis, S., Ebner, L., Christe, A., \& Mougiakakou, S. (2016). Lung pattern classification for interstitial lung diseases using a deep convolutional neural network. IEEE Transactions on Medical Imaging, 35(5), 1207-1216. doi:10.1109/TMI.2016.2535865 PMID:26955021

Swets, J. A., Dawes, R. M., \& Monahan, J. (2000). Better decisions through science. Scientific American, 283(4), 82-87. doi:10.1038/scientificamerican1000-82 PMID:11011389

Fawcett, T. (2006). An introduction to ROC analysis. Pattern Recognition Letters, 27(8), 861-874. doi:10.1016/j. patrec.2005.10.010

Lei Zhang received a Ph.D. in computer science from Harbin Institute of Technology, Harbin, China, in 2013. From September 2011 to August 2012, He was a research intern in Siemens Corporate Research, Inc., Princeton, NJ. From July 2015 to September 2017, He was a Post-Doctoral Research Fellow in College of Engineering, Temple University, PA and a Post-Doctoral Research Fellow in Department of Biomedical Informatics, Arizona State University, Scottsdale, AZ, respectively. He is currently a Postdoctoral associate in the School of Medicine, Department of Radiology at the University of Pittsburgh. He was a lecturer in School of Art and Design, Harbin University, Harbin, China. His current research interests include machine learning, computer vision, visualization and medical image analysis.

Qiufeng Wu was born at Heilongjiang Province, China, in 1979. He received Ph.D in computer application technology from Harbin Institute of Technology, Harbin, China, in January 2014. He is working as associated professor in College of Science in Northeast Agricultural University. He is CCF member. His current research interests include machine learning, computer vision and smart agriculture. 